\title{
Business Sentiment Analysis. Concept and Method for Perceived Anticipated Effort Identification
}

\author{
Nina Rizun \\ Gdansk University of Technology \\ Gdansk, Poland
}

Aleksandra Revina

Technical University of Berlin

Berlin, Germany nina.rizun@pg.edu.pl

revina@tu-berlin.de

\begin{abstract}
Representing a valuable human-computer interaction interface, Sentiment Analysis (SA) is applied to a wide range of problems. In the present paper, the researchers introduce a novel concept of Business Sentiment (BS) as a measurement of a Perceived Anticipated Effort (PAE) in the context of business processes (BPs). BS is considered as an emotional component of BP task contextual complexity perceived by a process worker after reading the task text. PAE is interpreted as a business process (BP) key performance indicator predicting urgency, criticality and complexity of the BP task processing. Using qualitative evaluation, the researchers proved the workability of both BS concept and its effective application method to measure PAE. As practical contributions of the research, quantitative support in a form of statistical reports and qualitative support in a form of task prioritization recommendations and time management for a BP worker are suggested.
\end{abstract}

Keywords: Sentiment Analysis, Business Sentiment, Perceived Anticipated Effort, Business Processes

\section{Introduction}

The roots of Sentiment Analysis (SA), also known as opinion mining, go back to the studies on public opinion at the beginning of the 20th century and the linguistic text subjectivity analysis in the 1990s. However, the topic continues to penetrate into new domains and application areas. In the scientific community, it is recognized to be one of the fastest growing research areas [21]. SA has been proven valuable for a wide range of problems, such as those coming from sociology, marketing, advertising, political sciences, representing one of the human-computer interaction interfaces [15].

SA allows automatic extraction of the knowledge about opinions, emotions, and attitudes hidden in the text. This determines the SA main application area - social media, i.e. understanding opinions of people about products, services, events, films, or politicians. Some novel SA applied research appeared in the areas of programming code reviews [24], cryptocurrencies [5], forecasting stock markets [28], or dynamic pricing models [43]. Nonetheless, such domain as business processes (BPs) in the context of SA has not been researched so far. There are considerable studies and tools related to the business reviews. However, they mainly address customer opinion mining regarding certain products and services to support decision-making, perform trend analysis or make predictions [36], [21].

In order to address this gap, the authors suggest a novel concept of sentiment - Business Sentiment (BS). With an increasing usage of diverse digital channels and resulting communication flows in enterprises, the amount of generated unstructured and semistructured data in the internal BPs is growing proportionally. This creates a demand for new qualitative and quantitative approaches, methods and tools to analyze these data [29], $[31,32]$. While observing the trend, the researchers made a conclusion that the SA of the internally generated unstructured BP textual data is determined by the business context specificity, i.e. such criteria as business ethics vocabulary or words indicating the 
complexity and resulting stress level of the BP task.

The remainder of the paper is organized as follows. Section 2 gives an overview of the related work, corresponding challenges, motivations, and derived research questions associated with SA and specifically BS. Section 3 presents the authors' proposed BS concept and method based on the case study data set. Section 4 describes a qualitative evaluation of the proposed approach, whereas Section 5 discusses scientific and practical contributions of the research, its limitations and future work.

\section{Related Work and Research Questions}

According to the subject of the research, the related work can be structured into the following categories: 1) SA approaches and 2) SA application areas. While there are many exhaustive studies on the SA [19], the goal of the authors is to provide a brief overview of acknowledged work and techniques in the field highlighting the related challenges, motivation behind the proposed research and deriving research questions (RQs). Shortly after, the methodology to address the posed RQs will be presented.

\subsection{Sentiment Analysis Approaches}

There are mainly two SA approaches extended by their possible combinations (hybrid SA): 1) lexicon-based and 2) Machine Learning (ML)-based (see Table 1). In addition, a separate classification related to both points, type of lexical features extraction, is included into the overview.

\section{Lexicon-based Sentiment}

Lexicon-based approach serves as a basis for a considerable number of studies [15], [42]. A sentiment lexicon represents a collection of lexical features (e.g., single words, n-grams, emoticons) that are labelled according to their semantic load as positive, negative or neutral [18]. The lexicon approach requires manual creation and validation of the semantically loaded features. However, the time and effort investment is compensated with the robustness of the method if compared to ML-based approaches. The researchers classify existing lexicon-based methods using following criteria: 1) lexicon construction methods and 2) categorization methods of lexical features. Below, a brief overview is presented.

In the lexicon construction methods, one can differentiate two basic approaches [19]: 1) based on thesaurus compiled by experts and 2) based on text corpus. Within the first one, a set of sentiment loaded lexical features (e.g., words) is collected and a seed list is manually created by the experts. Afterwards, the seeds are extended using existing online dictionaries (e.g., WordNet) to search for synonyms and antonyms. Disadvantage of thesaurus-based approach is that it requires certain manual effort for quality checking and, more important, does not function in case of finding domain or context-oriented sentiments. The corpus-based method addresses this disadvantage. Within this approach, a manually created seed list is extended with the corpus text what solves the problem of limited domain orientation. Owing to plentiful research contributions, several generalpurpose lexicons, such as General Inquirer [12], LIWC [39], SentiWordNet [4], SenticNet [9], ANEW [7], VADER [15], have been constructed and are widely used by the researchers leveraging applied SA. However, domain and context dependent sentiments remain to be problematic even with so much research on general-purpose sentiment lexicons [19].

In the methods of lexical features categorization, there is a following differentiation: 1) polarity-based (binary) categorization into positive and negative and 2) valence-based categorization in which lexical features are associated with valence scores for sentiment intensity. The first simple binary classification is known to yield more accurate results on single sentences [38]. However, the second approach while already reporting encouraging results is recognized to be more beneficial as it determines the strength of the sentiment expressed in text [15]. 


\section{Machine Learning-based Sentiment}

In the ML-based sentiment, the classification, supervised and unsupervised, is performed automatically while extracting features from text. Following the supervised approach, the sentiment classifier is trained using labelled training examples. Important supervised ML algorithms are Linear Classifiers (Support Vector Machine, Neural Network), Decision Trees, Rule-based and Probabilistic Classifier (Bayesian Network, Maximum Entropy, Naïve Bayes). Hereby, the biggest limitation is a high sensitivity to the quantity and quality of the training data, which can cause failure when training data is biased or insufficient [20]. This disadvantage is addressed by the unsupervised ML methods, such as LDA [6] or LSA [11], allowing to avoid the dependence on training the data. However, the limitation of unsupervised approaches is a large volume of data necessary for accurate training. Additionally, fully unsupervised models often produce incoherent results due to the lack of correlation with human judgements [20]. Besides, such methods require complex tuning for a given domain. For example, the method based on the LDA approach in its original form is not able to effectively detect topics and requires an adjustment of identified topics to the target set of contexts [33]. Semi-supervised approach that learns from both labelled and unlabelled data aims to leverage the disadvantages of its predecessors. It is a relatively new approach motivated by the lack of labelled data in real world applications [19]. However, the success of semi-supervised learning strongly depends on the correct assumptions in model implementation. If the assumption by the model is wrong, unlabelled data may decrease accuracy [26].

Table 1. Summary of Basic SA Approaches, Their Benefits and Limitations

\begin{tabular}{|c|c|c|}
\hline Approach & Benefits & Limitations \\
\hline \multicolumn{3}{|c|}{ Lexicon-based (construction methods) } \\
\hline Thesaurus-based & $\begin{array}{l}\text { robustness in the sense of general-purpose } \\
\text { applicability }\end{array}$ & $\begin{array}{l}\text { manual creation and validation, does not } \\
\text { function for context-oriented sentiments }\end{array}$ \\
\hline Corpus-based & $\begin{array}{l}\text { high quality in the sense of domain } \\
\text { specific applicability }\end{array}$ & manual creation and validation \\
\hline \multicolumn{3}{|c|}{ Lexicon-based (lexical features categorization) } \\
\hline Polarity-based & $\begin{array}{l}\text { accurate and widely recognized in } \\
\text { structuring texts into two categories }\end{array}$ & narrow scope bound to binary classification \\
\hline Valence-based & $\begin{array}{l}\text { additional information regarding sentiment } \\
\text { intensity and its changes }\end{array}$ & $\begin{array}{l}\text { sophisticated in terms of lexicon and rules } \\
\text { development, results interpretation }\end{array}$ \\
\hline \multicolumn{3}{|c|}{ Machine Learning-based } \\
\hline Supervised & $\begin{array}{l}\text { mature and successful automatic approach } \\
\text { to traditional topic classification }\end{array}$ & $\begin{array}{l}\text { manual labelling of the data, sensitivity to the } \\
\text { quantity and quality of the training data }\end{array}$ \\
\hline Unsupervised & no need to label the data & $\begin{array}{l}\text { large volume of data necessary for accurate } \\
\text { training }\end{array}$ \\
\hline Semi-supervised & $\begin{array}{l}\text { usage of both labelled and unlabeled data } \\
\text { to shrink manual work and improve } \\
\text { accuracy }\end{array}$ & $\begin{array}{l}\text { accuracy strongly depends on the model } \\
\text { assumptions }\end{array}$ \\
\hline
\end{tabular}

\section{Lexical Features Extraction}

SA task is a sentiment classification problem, whereby the first step is to select and extract lexical features from text. According to the type of lexical features extraction, the following approaches are possible [22], [2]: 1) n-grams, or consecutive $n$ terms, such as unigram for one word, bigram for two words, etc.; 2) parts-of-speech tagging, e.g. adjectives and adverbs are believed to hold most of the sentiments in text; 3) stemming, process of removing prefixes and suffixes, which can help in classification but sometimes leads to a decrease in classification accuracy; 4) stop words which provide no or little information about sentiments and can be removed; 5) conjunction handling, e.g. certain conjunction words like "but", "although", "however" can change the whole meaning of the sentence.

\subsection{Sentiment Analysis Application Areas}

Massive amounts of data produced in social media have led to a significant interest in analyzing blog posts, tweets, messages, or customer reviews for sentiment identification [23]. That is why 
social media is fairly considered to be one of the SA widely spread application areas. With the growing interest to SA, the range of its application areas is also increasing. Currently, the following key directions can be highlighted: 1) brand and customer management: SA of blogs, tweets and posts is widely used to analyze the brand image, track customer feedbacks and in development of automatic dialogue systems [30], [27], [13], [8]; 2) politics: there is an immense interest in tracking social media sentiment towards politicians, electoral issues, national and international events [1]; 3) public health: SA is especially applicable for detecting depressions [45], cyber-bulling [10], tracking well-being [37], developing social robots [14]; 4) education: diverse tutoring and evaluation systems benefit from SA identifying correctness of answers, emotional state of the participant or teaching quality [25]. While novel promising application areas, such as cryptocurrencies [5] or programming code reviews [24], keep emerging, the SA application in the BP context remains not studied.

Taking into account the discussions in Sections 1 and 2, one can highlight the following motivations for the proposed research: 1) increasing amount of digital communication channels and produced unstructured and semi-structured textual data in the internal BPs; 2) increase of sources with decision-making relevant information; 3) difficulty to search for and extract meaningful knowledge necessary for decision-making at the right time and in the right place; 4) technology advancements allowing to elaborate on new approaches, methods and tools to address mentioned challenges. Moreover, while conducting previous research work (see also Section 3) regarding the semantic nature of the BP activity [31] and stylistic patterns reflecting the readability of the BP textual requests containing those activities [32], the researchers were missing an important knowledge component, i.e. emotions and mental feelings the original author puts into the BP request, also known as sentiment. Hypothetically, this knowledge could help to derive such BP Key Performance Indicators (KPIs) as urgency, criticality of the request and its related complexity. As current paper attests, sentiment is not a universal approach and needs to be adjusted for successful implementation in individual domains. Thus, the authors conducted a solid literature review in the search for the relevant research work. However, a specific SA application in the BPs is not well presented. Considering discussed motivations, the following RQs have been arisen:

RQ1: Are business processes characterized with specific sentiment?

RQ2: What business process KPIs can be measured with BS?

RQ3: What SA approaches and particular rules can be used for the BS implementation?

In order to address the mentioned RQs, the authors designed the following methodology: elaboration of the BS concept and the method of its practical application to measure PAE (Section 3) and qualitative evaluation (Section 4). Such common methods of Information Systems research as case study, computational analyses, laboratory experiments, interviews, and observations support the research methodology [41], [44].

\section{Business Sentiment Concept and Method}

In the section, the authors introduce a business context specific sentiment, Business Sentiment, and a method of its practical application to measure business context specific Perceived Anticipated Effort. Under a classical sentiment, one understands an attitude or opinion towards something, mental feeling, emotions towards entities such as products, services, individuals, issues, events, topics, and their attributes [19]. A common definition of an anticipated effort is the degree to which physical or mental exertion seems to be needed (high) or not needed (low) [17]. In the business context, such factors as worker's responsibility feeling together with anticipated effort and real degree of complexity of the processes and related tasks are important indicators that, among others, can be measured by an emotional state of a worker, or Business Sentiment. Thus, the latter can be considered as a result of the perception of the BP nature (simple or complex in its execution) by a process worker. Under Perceived Anticipated Effort (PAE), the researchers understand the measure of the level of such factors as urgency, criticality and complexity of the task influencing its further execution. In this research, the approach of extracting a complex of mentioned latent factors from the unstructured BP textual requests and its formalization in a form of PAE qualitative value (high, medium, low) are proposed. 
Setting the background of the paper, the authors refer to already conducted research, which was based on the same case study from IT ticket domain. The goal was to assess the complexity of the IT ticket processing based on the incoming ticket description texts. The following indicators were suggested for complexity measurements and recommendations: 1) semantic nature of the activity reflecting the BP contextual complexity extracted from the ticket texts [31]; 2) stylistic patterns identifying the readability of the IT ticket texts and influencing the process worker's perception of the contextual complexity of the ticket processing (BP tasks) embedded in the ticket text [32]. In [31], the authors developed a specific parts-of-speech based semantic tagging approach, RTCC framework (nouns as BP Resources, verbs as Techniques, adjectives as Capacities, and adverbs as Choices) and drafted a set of indicators, or contextual variables, supporting the identification of Decision-Making Logic. In [32], the researchers studied in detail the criteria of stylistic formulation of BP requests (IT tickets). While conducting multiple experiments with the case study textual data, the authors detected stylistic patterns illustrating the transition from telegraphic style (describing simple, explicit requests) to verbose (describing complex requests). However, both investigations lack the consideration of important emotional aspect of BP request formulation.

Thus, BS is suggested as an instrument for measuring an additional PAE indicator as an emotional component of BP request (IT ticket) contextual complexity perceived by the process worker after reading the ticket text and affecting such process regulations as ticket prioritization and time management. Therefore, in the research context, PAE is considered as a business process KPI, which can be used for improving mentioned regulations. In the subsections below, the concept of BS Lexicon and a method of its practical application as an instrument to measure PAE are presented.

ITIL-based [3] Change Management (CHM) ticket process and related incoming ticket texts serve as a case study to prove the workability of the proposed method and to partially verify the results of the present research. The mentioned case study process relates to ITIL subject area "Service Transition", which objective is to build and deploy IT services. Hereby, CHM deals with the processing of so-called Requests for Change, tickets issued in order to add, modify or remove anything in the internal IT infrastructure of a company that could have an effect on IT services [16]. The ticket data set in focus of the research originates from the ticketing system of the CHM department of a big enterprise with more than 200,000 employees worldwide. The computational analyses and qualitative evaluation interviews were conducted at the Telekom Innovation Laboratories (T-Labs), Deutsche Telekom Research \& Development unit and affiliated institute at the Technical University of Berlin [40].

\subsection{Concept of Business Sentiment Lexicon}

While developing the concept of BS Lexicon, the authors considered the findings of the Section 2.1, i.e. benefits and limitations of existing SA approaches as well as specificity of business application area of the proposed research. Thus, the authors synergistically combined two lexicon-based approaches, corpus- and thesaurus-based ones, and amended the thesaurus building process with an unsupervised Machine Learning approach LD/SA [34] for the knowledge extraction and summarization purposes (see Fig.1). Due to the specificity of business context, single words and n-grams (bi- and three-grams) are selected as lexical features for lexicon development. 


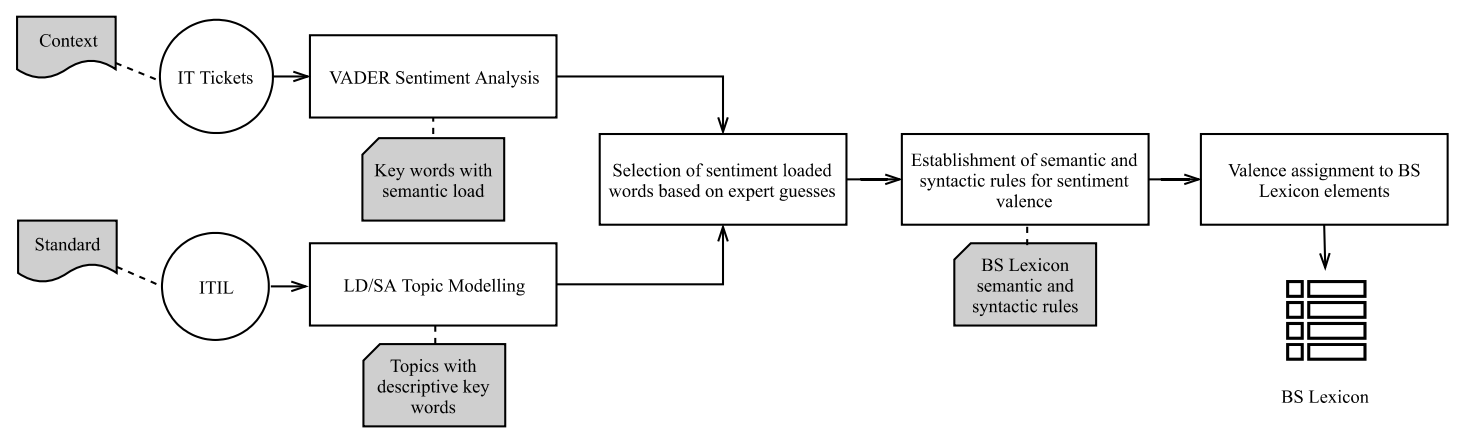

Fig.1. Business Sentiment Lexicon Development

As the data for the BS Lexicon development, two sources are used: 1) corpus, i.e. contextual ticket texts and 2) standard CHM related descriptions from ITIL Handbook. Accordingly, the following five steps were included in the concept development: 1) the researchers performed standard SA with the help of VADER [15] on the case study corpus (IT ticket texts). VADER approach (lexicon-/ thesaurus-based) was selected due to its general-purpose robustness and popularity as a gold standard list of lexical features, along with their associated sentiment intensity (valence); 2) based on the valence distribution for each ticket text entry in the corpus, key words with semantic load (negative and positive) were identified; 3) in parallel, the researchers extracted main topics with descriptive key words out of the standard ITIL CHM description texts while performing unsupervised LD/SA analysis; 4) using the contextual key words from the ticket texts extended with the standard ITIL key topics, the final list of lexical features was created. The process was supported by the method of expert guesses and a web conference interview with the case study process workers to validate the lexical features; 5) afterwards, considering the logic of VADER and business context, the researchers established a set of semantic and syntactic rules for BS analysis (Table 2).

Table 2. Comparative Rules of Valence

\begin{tabular}{|c|c|c|}
\hline VALENCE RULES & VADER [15] & BS \\
\hline Scoring rules & {$[-4 ;+4]$} & {$[-2 ;+2]$} \\
\hline \multicolumn{3}{|l|}{ Semantic rules } \\
\hline $\begin{array}{l}\text { Typical business ethics words (e.g., } \\
\text { "please", "dear", "thank you") }\end{array}$ & strongly positive & decreased to 0 \\
\hline $\begin{array}{l}\text { Words denoting complex IT problem solving } \\
\text { (e.g., "incident", "emergency", "downtime") }\end{array}$ & strongly negative & slightly increased to $-0.5 /-1$ \\
\hline $\begin{array}{l}\text { Typical daily work of IT ticket domain words } \\
\text { (e.g., "problem", "failed", "adequate") }\end{array}$ & positive/ negative & $\begin{array}{l}\text { categorized as neutral with } 0 \text { valence } \\
\text { as they belong to daily work }\end{array}$ \\
\hline $\begin{array}{l}\text { Typical positive words (e.g., "well", } \\
\text { "successful", "happy") }\end{array}$ & strongly positive & slightly decreased to +0.5 \\
\hline \multicolumn{3}{|l|}{ Syntactic rules (intensifiers) } \\
\hline Capitalizations & additional +0.733/-0.74 & additional +/- 0.5 \\
\hline “!”, “**”, “=”, “-”, “\#” & - & alone standing intensifier -0.1 \\
\hline Negation & regular negation words & "no", "not" \\
\hline
\end{tabular}

To sum up, the researchers build the BS Lexicon differentiating between three key words categories: 1) business ethics words, 2) standard ITIL CHM words and 3) corpusbased IT tickets. Based on the context independent semantic rules, the valence is assigned to the BS Lexicon elements. The rules of syntactic intensifiers are used while running the BS Lexicon on the case study data set.

\subsection{Method of Perceived Anticipated Effort Identification}

In the current section, the method of BS Lexicon practical application as an instrument to measure PAE is presented (see Fig. 2). The computational analyses of the present research 
were conducted in Python 3.4.3.

The experimental set-up includes the following six steps: 1) the researchers preprocessed the IT ticket texts removing stop words and performing stemming. The stemming step was performed in order to increase the match between ticket texts and BS Lexicon elements; 2) afterwards, the BS analysis was carried out; 3) based on the BS results, a compound score out of key word matches between IT ticket texts and the BS Lexicon was calculated. Hereby, the weighting of positive, neutral, negative was computed based on the valence scores assigned to the BS Lexicon elements in the concept building phase and adjusted with the syntactic rules (see Section 3.1); 4) as the next step, the summarization and interpretation rules of the obtained scores were developed; 5) in line with the traditional polarity-based SA and applying BS context logic, the relative values of binary positive-neutral (sum of both scores) and negative ticket scores were determined. As mentioned in Section 2.1, being one of the oldest and most traditional SA methods, polarity-based SA represents a solid approach of the corpora structuring; 6) based on the statistical distribution of compound valence scores and expert guesses, the following rules for PAE identification based on the valence score are accepted. Those tickets that are not recognized in the BS analysis contain prevailingly technical specifications (names of servers, databases, applications, etc.) and are written in a technically clear way. In this case, process workers already know what needs to be done. Therefore, the PAE of unrecognized tickets is assigned to be low (Table 3).

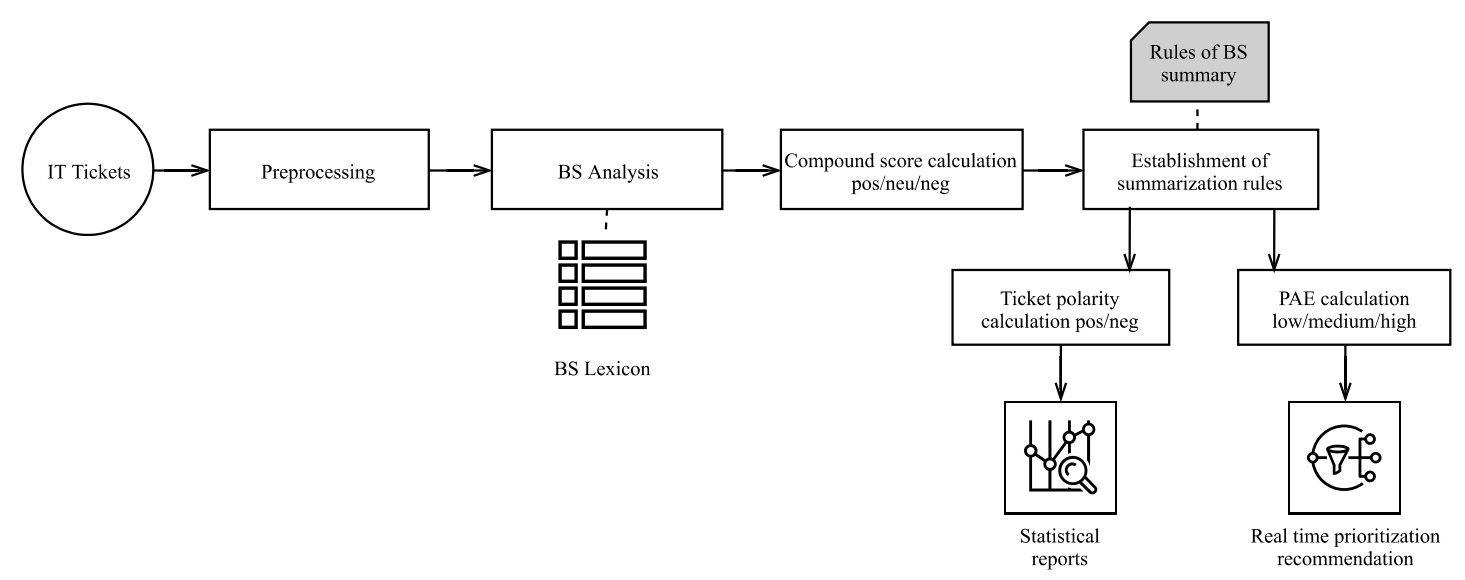

Fig.2. Experimental Set-Up of Method

Table 3. PAE Computation Rules

\begin{tabular}{|c|c|c|c|}
\hline \multicolumn{3}{|c|}{ INPUT } & OUTPUT \\
\hline \multicolumn{3}{|c|}{ Compound Valence (positive, neutral, negative) } & $\begin{array}{l}\text { Perceived Anticipated Effort } \\
\text { (low, medium, high) }\end{array}$ \\
\hline positive $>0.2$ & neutral $>2 *$ abs(negative) & $0<$ abs $($ negative $)<0.1$ & low \\
\hline positive $>=0$ & neutral $=0$ & negative $=0$ & low \\
\hline positive $>2 *$ neutral & neutral $>0$ & negative $=0$ & low \\
\hline \multicolumn{3}{|c|}{ unrecognized } & low \\
\hline positive $=0$ & neutral $=1$ & negative $=0$ & medium \\
\hline positive $=0$ & neutral $=0$ & negative $=0$ & medium \\
\hline positive $>0$ & neutral $>0$ & negative $=0$ & medium \\
\hline positive $>=0$ & neutral $>=0$ & $0<$ abs(negative) $<0.1$ & medium \\
\hline \multicolumn{3}{|c|}{ else } & high \\
\hline
\end{tabular}

PAE results should provide a real-time recommendation for a process worker in a form of correct prioritization of tasks in general and tickets in the particular context of the present research (qualitative analysis and recommendation). 


\section{Evaluation of the Business Sentiment Concept and Method}

In the evaluation phase, the researchers used a two-step approach: 1) comparing VADER and BS performance, 2) interviewing process workers. In the first step, the researchers aimed at detecting and statistically proving the difference in performance of suggested BS and a standard SA approach VADER. In the second step, the main goal was to determine to what extent the performance of BS vs standard SA approach (VADER) and the discrepancies identified in the first step correspond with the case study reality.

The evaluation data set of the case study was processed and converted into a CSV formatted text corpus with more than 1,000,000 documents (text entries) of English, German and mixed ticket texts created in the period of 2015-2018. After removing duplicates and selecting English texts, the final sample comprised 28,157 entries.

At the beginning, a preliminary calculation and assessment of the overall structure of the data set in the view of the research artefacts, i.e. polarity-based BS and PAE, was performed with the following statistical distributions: 1) polarity-based corpus structure: 69.02\% positive-neutral and 30.98\% negative tickets and 2) PAE identification: $8.7 \%$ tickets with low, $68.5 \%$ medium and $22.8 \%$ high PAE. The unrecognized part of the tickets with technical specification was assigned a low PAE (see Table 3).

As the first evaluation step, the comparison of VADER and BS (compound score) was performed (see Fig. 3 and Table 4). Hereby, the BS analysis yielded the following results: $8.76 \%$ positive scores, $64.49 \%$ neutral and $19.79 \%$ negative. The majority of neutral key words were identified based on the ITIL standard vocabulary. However, $6.96 \%$ of tickets were not recognized and therefore were categorized by the researchers as prevailingly containing technical specifications, i.e. names of servers, databases, applications, etc. To sum up, the analysis shows that BS is capturing more neutrality and significantly less positivity than VADER, which is also more characteristic for the business contexts. Furthermore, there are significant qualitative discrepancies in identification of negativity, which would be complaint with the business context as shown in Table 4.

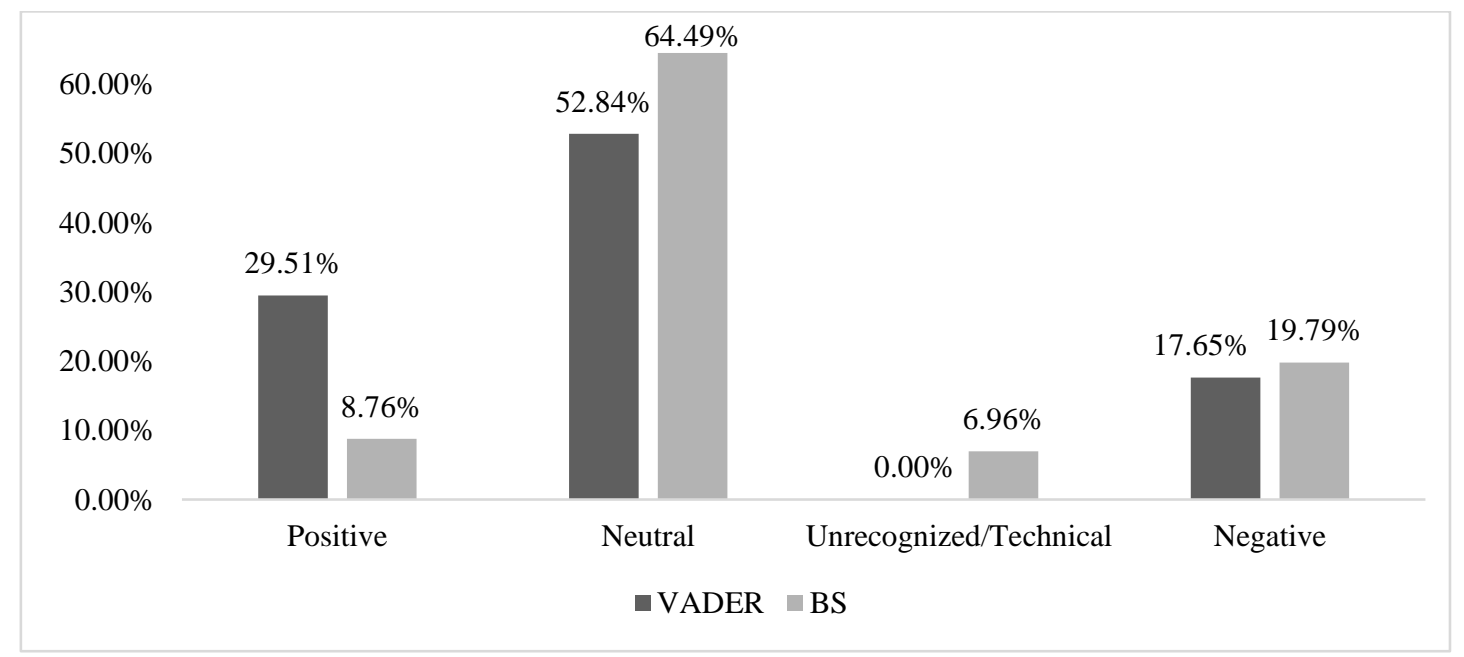

Fig. 3. Comparative Results VADER vs BS

Table 4. Comparative Results of Discrepancies VADER vs BS in \%

\begin{tabular}{|c|c|c|c|}
\hline Sentiment & $\begin{array}{c}\text { Discrepancies (+) } \\
\text { BS > VADER }\end{array}$ & $\begin{array}{c}\text { Discrepancies (-) } \\
\text { BS < VADER }\end{array}$ & Discrepancies (0) \\
\hline Positive & 7.21 & 49.15 & 43.64 \\
\hline Neutral & 38.24 & 40.06 & 21.70 \\
\hline Negative & 23.35 & 15.77 & 60.87 \\
\hline
\end{tabular}

To illustrate the performance of VADER vs BS, an exemplary anonymized ticket is presented in Table 5 (key words matched with VADER and BS Lexicon are marked grey). 
Table 5. Sentiment Analysis on Anonymized Ticket Example

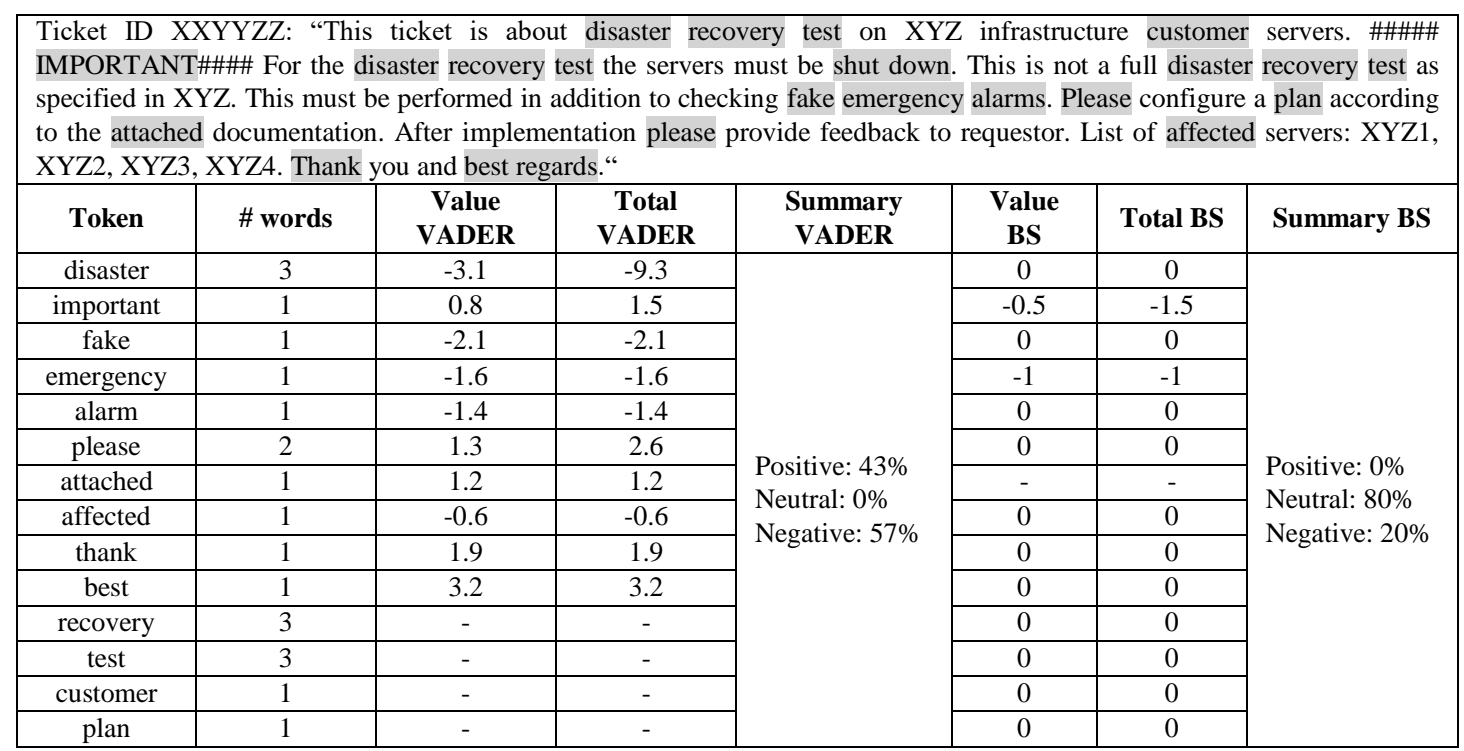

Applying the considerations mentioned in Section 3.1, one can clearly see that VADER approach is assigning highly negative values to such tokens as "disaster", "fake", "alarm" what contradicts the reality. These words belong to the specific IT domain vocabulary implying daily routine of a process worker. In contrast, BS Lexicon approach captures the values as neutral due to the mentioned fact. The same occurs with the positive values. Vader appoints positive values to the words belonging to the typical business ethics vocabulary, such as "thank", "best", "please". Additionally, the application of syntactic rules, or intensifiers, (column Total VADER and Total BS) increases the mentioned discrepancies (see Table 2 for the syntactic rules of intensifiers). Assumingly, there is a business rule of prioritization of complex and urgent tickets. Given that there are three tickets in the queue, the illustrated ticket will be assessed by BS approach as neutral with low PAE. If the other two tickets are assessed with higher PAE, they will be recommended as those to start with. As it can be seen from the example, VADER would misleadingly (out of context) classify the ticket as prevailingly negative (with high PAE) and prioritize it. Summing up, VADER outputs $43 \%$ positive and $57 \%$ negative scores and BS $80 \%$ neutral and $20 \%$ negative scores. These numbers clearly demonstrate that VADER outputs classical SA results that are misleading in the business context. In contrast, BS analysis correctly captures the emotional state of the worker as a part of its business environment considering such factors as daily business routine, task complexity and urgency.

As the second step in the evaluation process, the researchers introduced the overall BS concept and method as well as comparative results of VADER and BS performance to the case study process workers in terms of a web conference presentation followed by a Q\&A session supervised and executed by T-Labs. For this purpose, a semi-structured interview approach was selected with a planned set of questions regarding the feasibility and applicability of the BS vs VADER in identification of PAE. The interview session was divided into three parts. First, the researchers introduced the objectives of the interview, research motivations, theoretical and methodological background. Afterwards, the performance of both approaches was illustratively presented using a randomly selected IT ticket from the case study data set. Finally, a Q\&A session was conducted following a socalled funnel model [35], i.e. the researchers started with open questions and moved towards more specific ones regarding the practical value of the research artefacts. As compared to VADER, the BS approach was collectively evaluated as more feasible in the identification of request urgency, criticality, related task complexity, and respectively PAE. Moreover, process experts expressed their interest and willingness to incorporate the BS feature into the currently used ticketing system as a dashboard recommendation helping to prioritize the tickets. 


\section{Conclusion and Future Work}

In the present paper, the researchers introduced a novel concept and method of sentiment analysis, Business Sentiment. BS is suggested as an instrument for measuring Perceived Anticipated Effort in the BP task processing. Hereby, BS is interpreted as an emotional component of IT ticket contextual complexity perceived by a process worker after reading the ticket text. While developing BS concept and method, the authors leveraged the synergies of widely recognized SA approaches, such as lexicon-based (thesaurus and corpus) and unsupervised Machine Learning.

Thus, as scientific contributions of the research, the following can be acknowledged: 1) introduction of Business Sentiment as a novel sentiment type; 2) adaption of the PAE indicator concept to the BP context; 3) method of PAE measurement by Business Sentiment scores. These contributions allow giving positive answers to the research questions of the paper: 1) novel Business Sentiment concept expressing and capturing the specificity of the BP environment has the right to exist (RQ1); 2) presentation of the PAE concept measured by BS scores provides an answer to RQ2. Hereby, PAE serves as a BP KPI for process workers to make correct task prioritization and time management; 3 ) detailed description of the method of BS implementation, i.e. PAE identification, supported by the relevant state-of-the-art SA present a comprehensive answer to the RQ3, i.e. approaches and particular rules that can be used in the BS implementation.

As practical contributions of the research, the following two types of the support for the process workers can be determined: 1) quantitative support: BS polarity-based analysis is suggested to provide statistical reports on the BS identified in the corpus with the corresponding deep dives into the separate clusters. The actionable insights could be thought through recommendations for the team and department work, process optimization and automation measures; 2) qualitative support: based on the PAE calculated in real time, the process worker will get the priority recommendation of an incoming ticket in a form of a dashboard based on the established business rules. The mentioned dashboard will propose the right ticket order execution. Thus, the time is saved, the stress level decreases and the process worker can start with the ticket processing in a correct order, which is also beneficial for the business.

The researchers experienced the following limitations that will be addressed in the future work correspondingly: 1) limitations related to the validation of the BS results in PAE identification. The researchers plan to include other known process parameters such as timestamps or number of tasks related to the ticket;2) limitations related to the practical value demonstration of the research. Here, the authors plan to build a prototype of a Recommender System that will automatically extract information out of the incoming ticket textual requests and adapt the type and the way of recommendation based on the detected PAE and further criteria from the mentioned research of the authors.

\section{References}

1. Abdullah, M., Hadzikadic, M.: Sentiment Analysis of Twitter Data: Emotions Revealed Regarding Donald Trump during the 2015-16 Primary Debates. In: Proceedings of the 29th International Conference on Tools with Artificial Intelligence, pp. 760-764. IEEE, Boston (2017)

2. Adnan, D., Fei, S.: Feature Selection for Sentiment Analysis Based on Content and Syntax Models. Decision Support Systems 53, 704-711 (2012)

3. Axelos Global Best Practice. ITIL (2019), https://www.axelos.com/certifications/itilcertifications. Accessed March 28, 2019

4. Baccianella, S., Esuli, A., Sebastian, F.: SENTIWORDNET 3.0: An Enhanced Lexical Resource for Sentiment Analysis and Opinion Mining. In: Proceedings of LREC, pp. 2200-2204. European Language Resources Association, Malta (2010)

5. Bibi, S., Hussain, S., Faisal, M. I.: Public Perception Based Recommendation System for Cryptocurrency. In: Proceedings of the 16th International Bhurban Conference on Applied Sciences \& Technology, pp. 661-665. IEEE, Islamabad (2019)

6. Blei, D. M., Ng, A. Y., Jordan, M. I.: Latent Dirichlet Allocation. Journal of Machine 
Learning Research, 993-1022 (2003)

7. Bradley, M. M., Lang, P. J.: Affective Norms for English Words (ANEW): Instruction Manual and Affective Ratings. The Center for Research in Psychophysiology, University of Florida, Florida (1999)

8. Bröck, R., Glüge, S., Wendemuth, A., Limbrecht, K., Walter, S., Hrabal, D., Traue, H. C.: Intraindividual and Interindividual Multimodal Emotion Analyses in Human-MachineInteraction. Cognitive Methods in Situation Awareness and Decision Support, pp. 59-64. IEEE, New Orleans (2012)

9. Cambria, E., Havasi, C., Hussain, A.: SenticNet 2: A Semantic and Affective Resource for Opinion Mining and Sentiment Analysis. In: Proceedings of the 25th International Florida Artificial Intelligence Research Society Conference, pp. 202-207. AAAI Press, Marco Island (2012)

10. Chen, Y., Zhou, Y., Xu, H.: Detecting Offensive Language in Social Media to Protect Adolescent Online Safety. In: Proceedings of International Conference on Social Computing (SocialCom), pp. 71-80. IEEE, Amsterdam (2012)

11. Dumais, S. T., Furnas, G. W., Landauer, T. K., Deerwester, S.: Using Latent Semantic Analysis to Improve Information Retrieval. In: Proceedings of the 88th Conference on Human Factors in Computing, pp. 281-285. ACM, New York (1988)

12. Green, B. F., Stone, P. J., Dunphy, D., Smith, M. S., Ogilvie, D. M.: The General Inquirer: A Computer Approach to Content Analysis. MIT Press, Cambridge (1966)

13. Gupta, N., Gilbert, M., Di Fabbrizio, G.: Emotion Detection in Email Customer. Computational Intelligence 29 (3), 489-505 (2013)

14. Hossain, Y., Hossain, I., Banik, M., Hossain, I., Chakrabarty, A.: Embedded System based Bangla Intelligent Social Virtual Robot with Sentiment Analysis. In: Proceedings of Joint 7th International Conference on Informatics, Electronics \& Vision, pp. 322-327. IEEE, Kitakyushu (2018)

15. Hutto, C. J., Gilbert, E.: VADER: A Parsimonious Rule-based Model for Sentiment Analysis of Social Media Text. In: Proceedings of the 8th International Conference on Weblogs and Social Media. AAAI, Ann Arbor (2014)

16. ITIL ${ }^{\circledR}$ Service Transition. TSO, London (2011)

17. Lerner, J. S., Li, Y., Valdesolo, P., \& Kassam, K. S.: Emotion and Decision Making. Annual Review of Psychology 66, 799-823 (2015)

18. Liu, B.: Sentiment Analysis and Subjectivity. In: Indurkhya, N., Damerau, F. J. (eds.) Handbook of Natural Language Processing, pp. 1-38. CRC Press, Boca Raton (2010)

19. Liu, B.: Sentiment Analysis and Opinion Mining. Morgan \& Claypool Publishers, San Rafael (2012)

20. Madhoushi, Z., Hamdan, A. R., \& Zainudin, S.: Sentiment Analysis Techniques in Recent Works. In: Proceedings of the Science and Information Conference, pp. 288-291. IEEE, London (2015)

21. Mäntylä, M. V., Graziotin, D., \& Kuutila, M.: The Evolution of Sentiment Analysis - A Review of Research Topics, Venues, and Top Cited Papers. Computer Science Review 27, 16-32 (2018)

22. Mejova, Y., \& Srinivasan, P.: Exploring Feature Definition and Selection for Sentiment Classifiers. In: Proceedings of the 5th International Conference on Weblogs and Social Media, pp. 546-549. AAAI Press, Barcelona (2014)

23. Mohammad, S. M.: Sentiment Analysis: Detecting Valence, Emotions, and Other Affectual States from Text. In: Meiselman, H. L. (ed.) Emotion Measurement, pp. 201 237. Woodhead Publishing, London (2016)

24. Paul, R., Bosu, A., Sultana, K. Z.: Expressions of Sentiments during Code Reviews: Male vs. Female. In: Proceedings of the 26th International Conference on Software Analysis, Evolution and Reengineering, pp. 26-37. IEEE, Hangzhou (2019)

25. Pong-Inwong, C., Kaewmak, K.: Improved Sentiment Analysis for Teaching Evaluation Using Feature Selection and Voting Ensemble Learning Integration. In: Proceedings of the 2nd International Conference on Computer and Communications, pp. 318-323. IEEE, Chengdu (2016)

26. Prakash, J., Nithya, L.: A Survey on Semi-Supervised Learning Techniques. International 
Journal of Computer Trends and Technology 8(1), 25-29 (2014)

27. Ren, F., Quan, C.: Linguistic-based Emotion Analysis and Recognition for Measuring Consumer Satisfaction: an Application of Affective Computing. Information Technology and Management 13(4), 321-332 (2012)

28. Ren, R., Wu, D. D., Liu, T.: Forecasting Stock Market Movement Direction Using Sentiment Analysis and Support Vector Machine. IEEE Systems Journal 13(1), 760-770 (2019)

29. Revina, A.: Assessing Process Suitability for AI-based Automation. In: Business Information Systems Workshops (BIS 2018), Lecture Notes in Business Information Processing 339, pp. 697-706. Springer, Berlin (2019)

30. Rizun, N., Kucharska, W.: Text Mining Algorithms for Extracting Brand Knowledge. The Fashion Industry Case. In: 31st International Business Information Management Association (IBIMA), pp. 1972-1983. Web of Science, Milan (2018)

31. Rizun, N., Revina, A., Meister, V.: Method of Decision-Making Logic Discovery in the Business Process Textual Data. In: 22nd International Conference on Business Information Systems (BIS 2019). Springer, Seville (2019)

32. Rizun, N., Revina, A., Meister, V.: Discovery of Stylistic Patterns in Business Process Textual Descriptions: IT Ticket Case. In: 33rd International Business Information Management Association (IBIMA). Web of Science, Granada (2019)

33. Rizun, N., Taranenko, Y., Waloszek, W.: The Algorithm of Modelling and Analysis of Latent Semantic Relations: Linear Algebra vs. Probabilistic Topic Models. In: International Conference on Knowledge Engineering and the Semantic Web, pp. 53-68. Springer, Szczecin (2017)

34. Rizun, N., Taranenko, Y., Waloszek, W.: Improving the Accuracy in Sentiment Classification in the Light of Modelling the Latent Semantic Relations. Information (Switzerland) 9(12), 53-68 (2018)

35. Runeson, P., Höst, M.: Guidelines for conducting and reporting case study research in software engineering. Empirical Software Engineering 14, 131-164 (2009)

36. Salinca, A.: Business Reviews Classification Using Sentiment. In: Proceedings of the 17th International Symposium on Symbolic and Numeric Algorithms for Scientific Computing, pp. 247-250. IEEE, Timisoara (2016)

37. Schwartz, A. et al.: Characterizing Geographic Variation in Well-Being Using Tweets. In: Proceedings of the 7th International AAAI Conference on Weblogs and Social Media, pp. 583-591. IEEE, Cambridge (2013)

38. Socher, R., Perelygin, A., Wu, J., Chuang, J., Manning, C., Ng, A., \& Potts, C.: Recursive Deep Models for Semantic Compositionality over a Sentiment Treebank. In: Proceedings of the Conference on Empirical Methods in Natural Language Processing, pp. 1631-1642. ACL, Washington (2013)

39. Tausczik, Y. R., \& Pennebaker, J. W.: The Psychological Meaning of Words: LIWC and Computerized Text Analysis Methods. Journal of Language and Social Psychology 29(1), 24-54 (2010)

40. Telekom Innovation Laboratories (2018), https://laboratories.telekom.com/. Accessed April 5, 2019

41. Wilde, T., Hess, T.: Forschungsmethoden der Wirtschaftsinformatik. Eine empirische Untersuchung. Wirtschaftsinformatik 49(4), 280-287 (2007)

42. Xiong, G., Fang, Y., Liu, Q.: Automatic Construction of Domain-specific Sentiment Lexicon Based on the Semantics Graph. In: Proceedings of the International Conference on Signal Processing, Communications and Computing, pp. 1-6. IEEE, Xiamen (2017)

43. Zhao, L. A.: Dynamic Pricing Mechanism Model Based on Sentiments Analysis. In: Proceedings of the International Conference on Intelligent Transportation, Big Data \& Smart City, pp. 622-625. IEEE, Changsha (2019)

44. Zimmermann, E.: Das Experiment in den Sozialwissenschaften. VS Verlag für Sozialwissenschaften, Wiesbaden (2008)

45. Zucco, C., Calabrese, B., Cannataro, M.: Sentiment Analysis and Affective Computing for Depression Monitoring. In: Proceedings of the International Conference on Bioinformatics and Biomedicine, pp. 1988-1995. IEEE, Kansas City (2017) 